\title{
Analyses of key gene networks controlling carotenoid metabolism in Xiangfen 1 banana
}

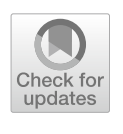

Chen Dong ${ }^{\dagger}$, Jiuxiang Wang ${ }^{\dagger}$, Yulin Hu, Weijun Xiao, Huigang Hu* and Jianghui Xie*

\begin{abstract}
Background: Banana fruits are rich in various high-value metabolites and play a key role in the human diet. Of these components, carotenoids have attracted considerable attention due to their physiological role and human health care functions. However, the accumulation patterns of carotenoids and genome-wide analysis of gene expression during banana fruit development have not been comprehensively evaluated.

Results: In the present study, an integrative analysis of metabolites and transcriptome profiles in banana fruit with three different development stages was performed. A total of 11 carotenoid compounds were identified, and most of these compounds showed markedly higher abundances in mature green and/or mature fruit than in young fruit. Results were linked to the high expression of carotenoid synthesis and regulatory genes in the middle and late stages of fruit development. Co-expression network analysis revealed that 79 differentially expressed transcription factor genes may be responsible for the regulation of LCYB (lycopene $\beta$-cyclase), a key enzyme catalyzing the biosynthesis of $a$ - and $\beta$-carotene.
\end{abstract}

Conclusions: Collectively, the study provided new insights into the understanding of dynamic changes in carotenoid content and gene expression level during banana fruit development.

Keywords: Banana, Developmental period, Carotenoid compounds, Gene networks

\section{Background}

Banana fruits play a key role in the human diet due to their desirable palatability and high nutritional value [1, 2]. Bananas are rich in various metabolites, such as soluble sugars, vitamins, carotenoids, phenolics, and minerals [3]. Of these components, carotenoids represent a large and diverse class of biological compounds and fulfill many important physiological functions [4]. However, the mechanism underlying carotenoid biosynthesis in banana remains unclear. Carotenoids in plants can produce a series of compounds named apocarotenoids under

\footnotetext{
*Correspondence: huhuigang@sina.com; 313972374@qq.com ${ }^{\dagger}$ Chen Dong and Jiuxiang Wang contributed equally to this work. South Subtropical Crop Research Institute, Chinese Academy of Tropical Agricultural Science/ Key Laboratory of Tropical Fruit Biology, Ministry of Agriculture/Key Laboratory of Hainan Province for Postharvest Physiology and Technology of Tropical Horticultural Products, Zhanjiang, Guangdong 524091, China
}

oxidative cleavage, which confers volatile compounds to the aromatic components of flowers, leaves, and fruits, as well as the well-known phytohormones, such as abscisic acid and strigolactones [5]. Carotenoids are typically tetraterpene (C40) molecules with 40 carbon atoms and multiple conjugated double bonds [6]. These bonds enable carotenoids in the selective absorption of certain wavelengths of the visible light spectrum to give bright colors, such as yellow, orange, and red, to fruits, flowers, and vegetables $[7,8]$. Thus, carotenoids have been as dyes for various industrial applications due to this property. Furthermore, carotenoids can serve as precursors for the biosynthesis of vitamin A and also provide precursors to many flavor-related compounds, which confer sensory attributes to the consumers [9]. Carotenoids have been used for the food, nutraceutical, and pharmacological industries due to their various beneficial effects on human and animal health [10]. 
Similar to other isoprenoids, carotenoids are synthesized via successive condensations of the five-carbon molecule isopentenyl diphosphate (IPP) and its isomer dimethylallyl diphosphate (DMAPP) [11] . Plants have two distinct routes for IPP and DMAPP biosynthesis: the cytosolic mevalonic acid and the plastid methylerythritol 4-phosphate pathways [12, 13]. Geranylgeranyl pyrophosphates (GGPP) are formed by three IPP and one DMAPP in plastids. First, the colorless carotenoid phytoene is formed by the condensation of two molecules of GGPP. Then, colorless phytoene is converted into red lycopene via a series of desaturation and isomerization. Lycopene can produce a large variety of carotenoids with different physical properties via various end-group modifications, such as $\alpha$-carotene, $\beta$-carotene, zeaxanthin, and lutein $[7,14]$. In addition to the structural genes, some transcription factors have been reported to be involved in the synthesis of carotenoids by regulating the expression of carotenoid biosynthetic genes, such as MADS-box [15], SBP-box [16], NAC [17], AP2/ERF [18], MYB [19], HD-Zip [20], and NF-Y [21].

Integrative analysis of metabolome and transcriptome profiles has been performed because the accumulation of metabolites is preceded by coordinated increases in the transcriptional level of relevant genes. Based on the correlation, this method has been widely applied to fig [22]., asparaguses [23], peach [24], ginkgo biloba [25], kiwifruit [26], and other plants. Nevertheless, integrated investigations on carotenoid biosynthesis characteristics and regulators are relatively few. Xiangfen 1, a novel flavonoid-rich banana germplasm, was used in this study to perform the dynamic metabolites and transcriptome analyses in banana pulp at three different developmental stages and identify the accumulation patterns of carotenoids and their underlying regulation. An understanding of dynamic changes in carotenoid content and the gene expression level during fruit development is essential for the breeding of special banana subgroups with high carotenoid contents.

\section{Results \\ Variations among carotenoid content during banana fruit flesh development}

As shown in Fig. 1, 11 carotenoid compounds, including $\alpha$-carotene, antheraxanthin, violaxanthin, $\gamma$-carotene, neoxanthin, $\beta$-carotene, lutein, $\beta$-cryptoxanthin, $\beta$-apocarotenal, (E/Z)-phytoene, and $\alpha$-cryptoxanthin, were identified from the banana pulp at different developmental stages. Most of the carotenoid compounds, such as $\alpha$-carotene, $\beta$-carotene, $\gamma$-carotene, $(\mathrm{E} / \mathrm{Z})$-phytoene, $\alpha$-cryptoxanthin, $\beta$-cryptoxanthin, and $\beta$-apocarotenal were undetectable or at considerably low levels at young fruits but substantially increased at mature green and/or mature fruits $(P<0.05)$. Interestingly, the highest level of violaxanthin was observed at young fruits and then gradually decreased with fruit development $(P<0.05)$.
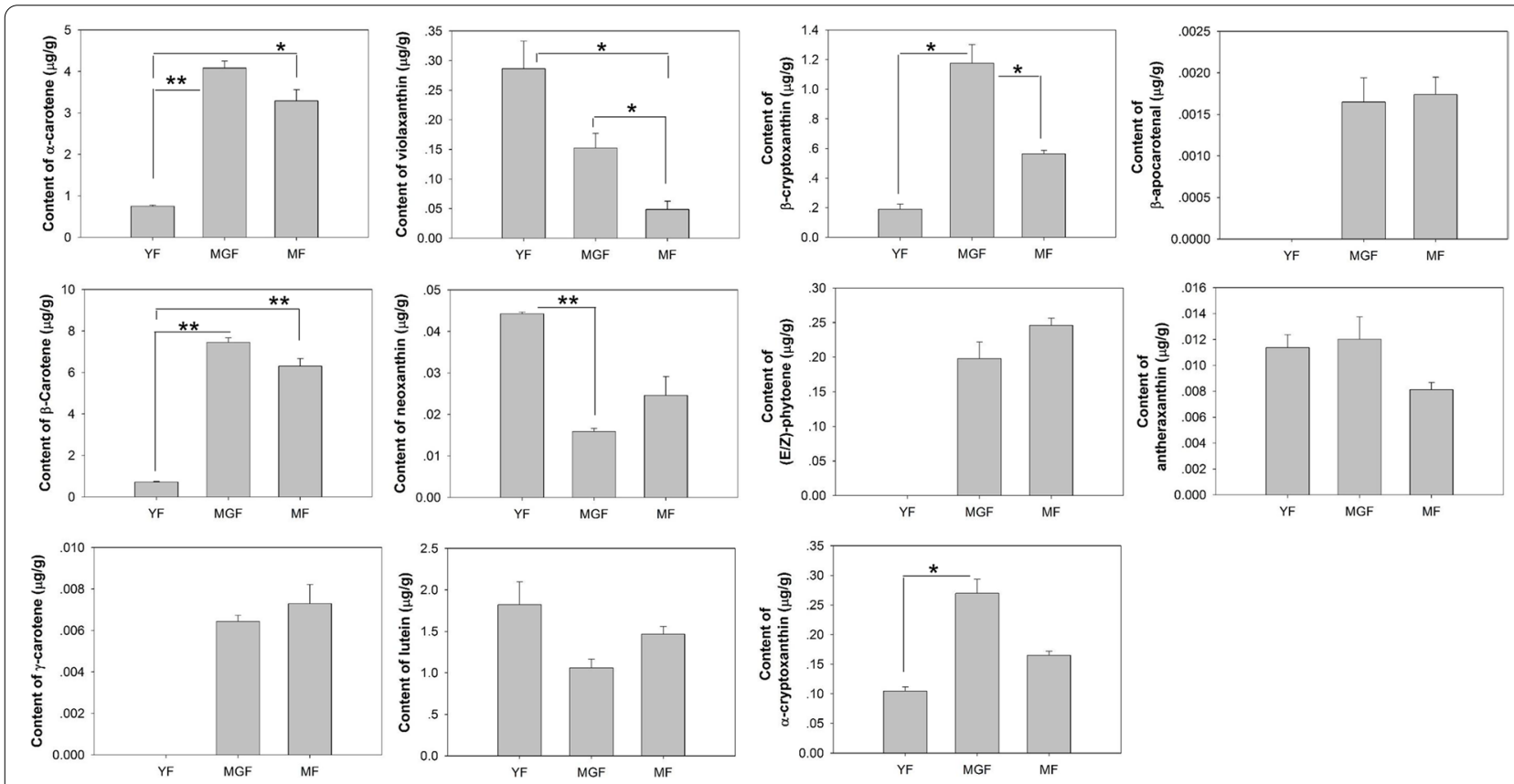

Fig. 1 Carotenoid content $(\mu \mathrm{g} / \mathrm{g})$ of banana pulp across three developmental stages 


\section{Identification of differentially expressed genes (DEGs)}

Using a $\mid \log _{2}$ fold change $\mid$ of $\geq 1$ and an FDR of $\leq 0.05$ as the thresholds, a total of 4590 (1703 upregulated and 2887 downregulated), 14,149 (6207 upregulated and 7942 downregulated) and 15,991 (6782 upregulated and 9209 downregulated) differentially expressed genes (DEGs) were identified in the three comparison groups: young and mature green, mature green and mature, and young and mature fruits, respectively. The majority of DEGs were downregulated during fruit development (Fig. 2A). The Venn diagram showed that 2703, 3737, and 12,195 DEGs were shared by two comparison groups, and 2205 DEGs were common to all three comparison groups (Fig. 2B).

\section{Enrichment of GO terms and KEGG pathway analysis}

Gene Ontology (GO) term analysis was assigned to the identified DEGs to evaluate the gene expression of fruit development (Fig. 3A, B, C). GO analysis classified $18,839,17,800$, and 17,469 genes into the biological process, cell component, and molecular function, respectively. Among the biological process categories, the cellular and metabolic processes account for a higher proportion, followed by biological regulation, response to stimulus, and regulation of biological process. The most highly represented terms within the cellular component categories were the cell, cell part, organelle, membrane, and membrane part. Meanwhile, the most highly represented terms in the molecular function categories included binding, catalytic activity, and transcription regulator activity.

The DEGs were mapped to the reference pathways in the KEGG database to obtain additional information regarding the biological pathways activated in the regulation of fruit development. Among the DEGs assigned to 309 KEGG pathways in the pairwise comparisons of the young fruit versus mature green fruit (Fig. 3D), the most highly enriched pathways included biosynthesis of secondary metabolites (ko01110, $P=2.6 \times 10^{-12}, 297$ genes), metabolic pathways (ko01100, $P=1.6 \times 10^{-9}$, 485 genes), plant hormone signal transduction (ko04075, $P=3.6 \times 10^{-6}, 97$ genes), bile secretion (ko04976, $P=3.5 \times 10^{-5}, 20$ genes), and phenylpropanoid biosynthesis (ko00940, $P=4.5 \times 10^{-5}, 52$ genes). A comparison of the DEGs between mature green and mature fruits resulted in the identification of 301 KEGG pathways (Fig. 3E). The most highly enriched pathways were arachidonic acid metabolism (ko00590, $P=6.18 \times 10^{-5}, 20$ genes), endocrine and other factor-regulated calcium reabsorption (ko04961, $P=2.9 \times 10^{-4}, 39$ genes), plant hormone signal transduction (ko04075, $P=2.4 \times 10^{-3}$, 239 genes), thyroid hormone signaling pathway (ko04919, $P=5.8 \times 10^{-3}, 34$ genes), and vasopressinregulated water reabsorption (ko04962, $P=0.01,41$ genes). In the comparison of young and mature fruits (Fig. 3F), DEGs were most highly enriched in endocrine and other factor-regulated calcium reabsorption (ko04961, $P=1.5 \times 10^{-4}, 43$ genes), pancreatic secretion (ko04972, $P=4.1 \times 10^{-4}, 45$ genes), biosynthesis of
A

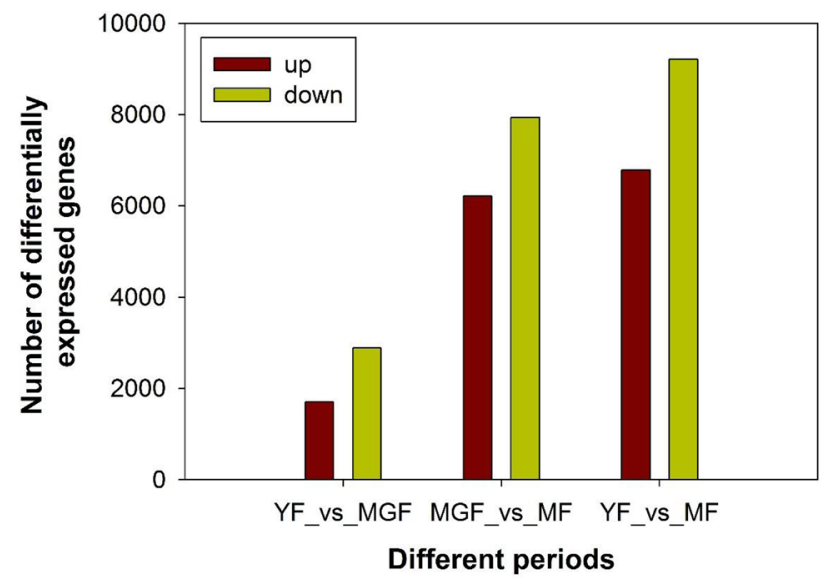

B

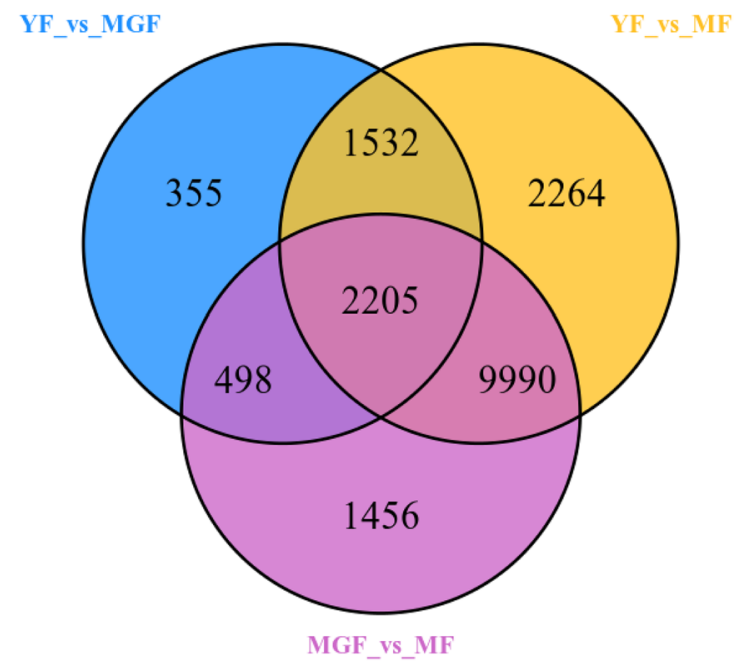

Fig. 2 Summary of differentially expressed genes (DEGs) during fruit development. A Numbers of DEGs. The numbers of up-regulated genes and down-regulated genes for each comparison group are indicated with red and yellow color, respectively. B A Venn diagram showing the overlapping and sample-specific DEGs from the young fruit, mature green fruit, and mature fruit 


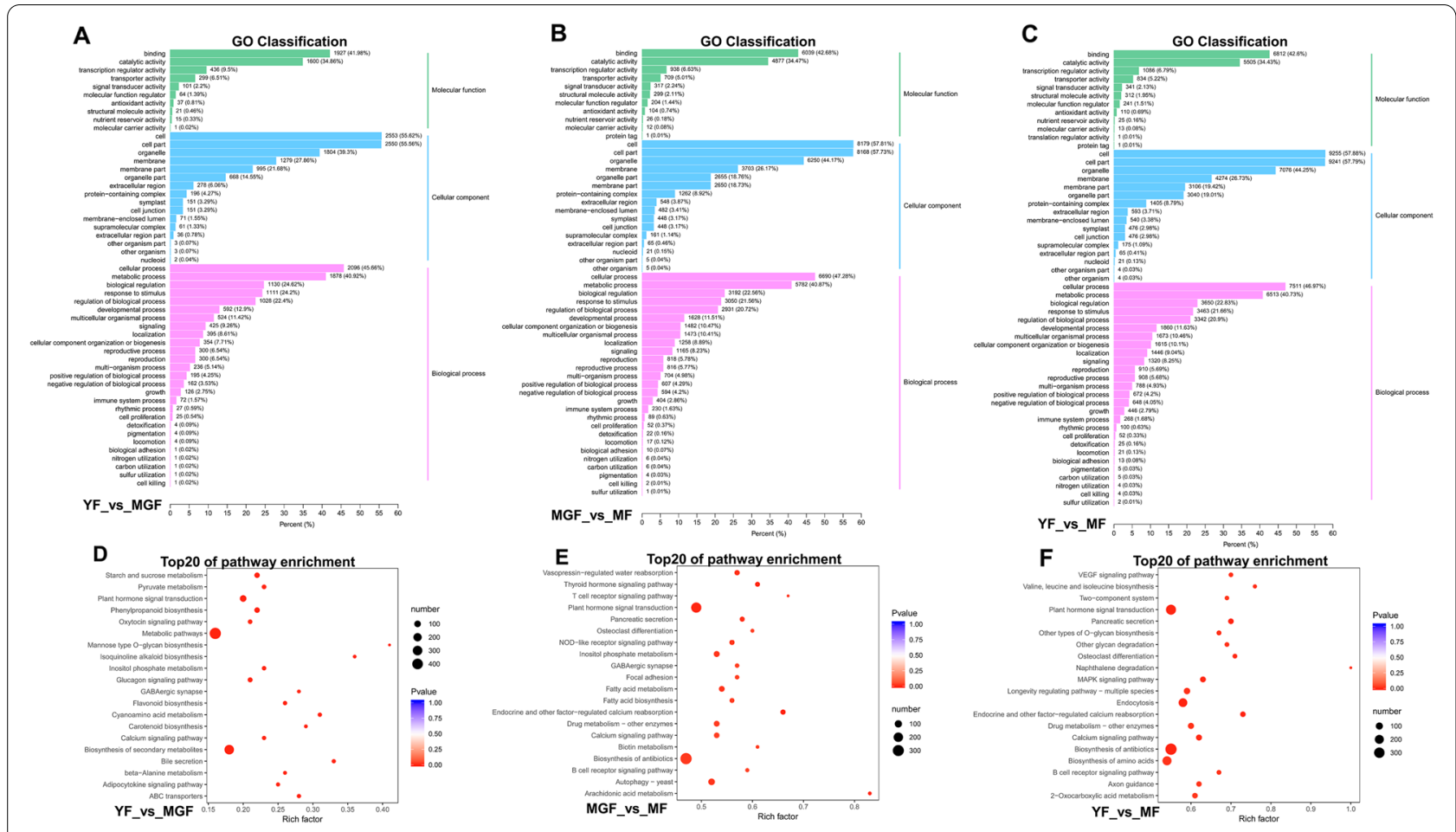

Fig. 3 Gene-ontology (GO) classification and Kyoto Encyclopedia of Genes and Genomes (KEGG) pathway analysis of DEGs during fruit development. Functional classification of DEGs based on GO between the young fruit and mature green fruit (A), mature green fruit and mature fruit (B), and young fruit and mature fruit (C), respectively. KEGG pathway analysis of DEGs for the young fruit versus mature green fruit (D), mature green fruit versus mature fruit $(\mathbf{E})$, and young fruit versus mature fruit $(\mathbf{F})$

antibiotics (ko01130, $P=6.6 \times 10^{-4}, 363$ genes), endocytosis (ko04144, $P=8 \times 10^{-4}, 182$ genes), and plant hormone signal transduction (ko04075, $P=2.7 \times 10^{-3}, 267$ genes). Notably, the comparison of young and mature green fruits revealed that the carotenoid biosynthesis (ko01130, $P=3.5 \times 10^{-3}$, 13 genes) was also enriched.

\section{Expression of genes related to carotenoid biosynthesis}

Carotenoid concentration is one of the main features that give an esthetic and nutritional value to banana fruit. Seven DEGs representing six genes were involved in carotenoid biosynthesis in banana in this study. The expression analysis of these DEGs is displayed in Fig. 4. The expression level of two genes encoding CRTB gradually decreased with fruit development, whereas the gene encoding Z-ISO, LCYB, LCYE, and CRTZ gradually increased during fruit development. The gene encoding VDE demonstrated high expression levels in the young fruit and low expression levels in the mature green and mature fruits.

\section{Transcription factors involved in carotenoid biosynthesis} Gene expression in plant carotenoid biosynthesis is strictly controlled by transcription factors. A total of 646 differentially expressed transcription factor genes were identified between the young and mature green fruits. Among these genes, 170 transcription factor genes were assigned to MADS-box (4 upregulated and 9 downregulated), SBP-box (0 upregulated and 13 downregulated), NAC (11 upregulated and 20 downregulated), AP2/ERF (16 upregulated and 29 downregulated), MYB (17 upregulated and 43 downregulated), and NF-Y (3 upregulated and 5 downregulated). Interestingly, most of the transcription factor genes demonstrated downregulation between the young and mature green fruits (Table 1).

\section{Co-expression network analysis of metabolites, genes, and transcription factors related to carotenoid biosynthesis}

A correlation network was constructed combining 10 metabolites, 7 enzyme genes, and 108 transcription factors related to carotenoid biosynthesis. Only the correlation pairs with a Pearson correlation coefficient $>0.8$ were included in this analysis (Fig. 5). The visualized network in Cytoscape showed that a total of 125 nodes were connected, linked by 910 edges. The gene-to-gene FPKM value and gene-to-metabolite accumulation pattern 


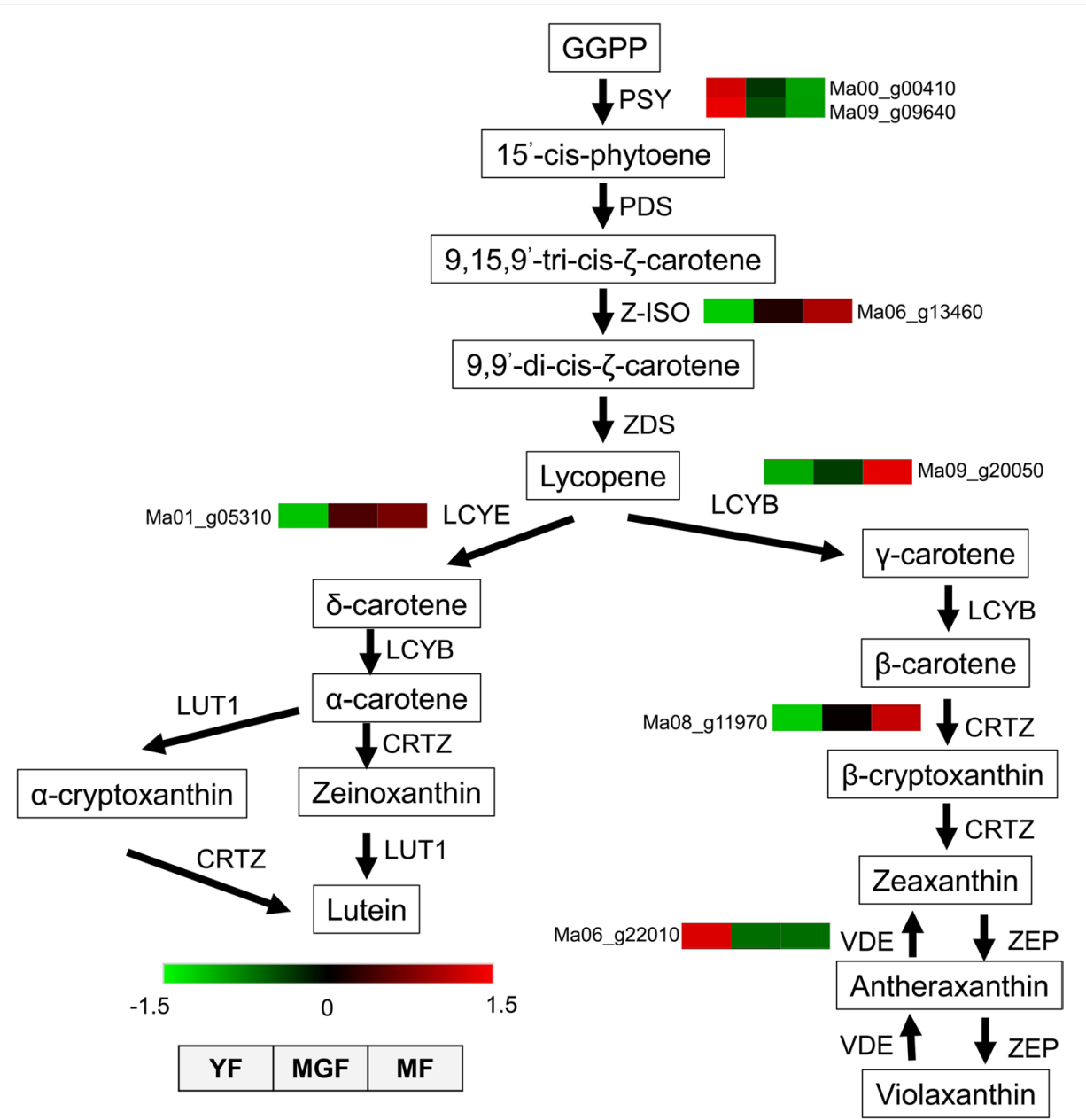

Fig. 4 Schematic presentation of carotenoid biosynthesis. The dashed arrows represent multiple enzymatic steps. The quadrates marked with green and red background represent the reduced and increased abundances of DEGs, respectively. PSY, phytoene synthase; PDS, phytoene

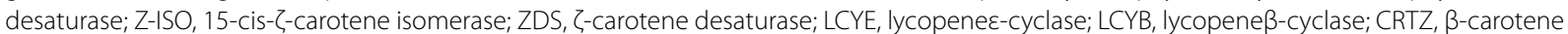
3-hydroxylase; VDE, violaxanthin de-epoxidase; ZEP, zeaxanthin epoxidase; LUT1, carotenoid epsilon hydroxylase

revealed that 351 and 559 pairs of nodes respectively showed positive and negative correlations.

Lycopene $\beta$-cyclase (LCYB) is a key enzyme catalyzing the biosynthesis of $\alpha$-carotene and $\beta$-carotene. In Fig. 5 , 79 (15 upregulated and 64 downregulated) differentially expressed transcription factor genes were filtered by direct correlation with the gene encoding LCYB.

\section{Validation of transcriptomic data by quantitative real-time PCR (qRT-PCR)}

A total of 23 DEGs (5 carotenoid biosynthetic pathway genes, 18 transcription factor genes) were used to analyze their expression levels in YF (young fruit), MGF (mature green fruit), and MF (mature fruit) using RT-qPCR to validate the key RNA-Seq results. The expression patterns of these genes were similar to the RNA-Seq results, with correlation coefficients $\left(R^{2}\right)>0.91$ (Fig. 6). The results validated the relevance of the RNA-Seq data, and RT-qPCR showed good consistency for upregulated and downregulated gene expressions.

\section{Discussion}

Carotenoids are widely distributed secondary metabolites that are not only crucial in plant physiology but also beneficial to human health as dietary components [27]. A total of 18 carotenoids were detected by the LC-MS/ MS in the present study to investigate the accumulation pattern of carotenoids during the entire developmental period of fruit. However, seven carotenoids remained undetected in this study due to the lower carotenoid content in the sample than the detection limit of the instrument or the absence of carotenoid in the sample. A previous study revealed that $\alpha$-carotene, $\beta$-carotene, and lutein displayed a dramatic increase with banana fruit 
Table 1 Transcription factors involved in carotenoid biosynthesis

\begin{tabular}{|c|c|c|c|c|c|}
\hline Number & Gene ID & Family & $\begin{array}{l}\text { Young fruit } \\
\text { Expression level }\end{array}$ & $\begin{array}{l}\text { Mature green fruit } \\
\text { Expression level }\end{array}$ & regulated \\
\hline 1 & Ma02_g02200 & MADS-M-type & 1096 & 424 & down \\
\hline 2 & Ma02_g12050 & MADS-MIKC & 8 & 165 & up \\
\hline 3 & Ma03_g08420 & MADS-M-type & 175 & 2 & down \\
\hline 4 & Ma03_g26480 & MADS-MIKC & 237 & 82 & down \\
\hline 5 & Ma03_g31640 & MADS-MIKC & 325 & 1157 & up \\
\hline 6 & Ma04_g30020 & MADS-MIKC & 5289 & 2312 & down \\
\hline 7 & Ma06_g01760 & MADS-MIKC & 258 & 92 & down \\
\hline 8 & Ma07_g00440 & MADS-MIKC & 122 & 14 & down \\
\hline 9 & Ma07_g25120 & MADS-MIKC & 476 & 75 & down \\
\hline 10 & Ma08_g04740 & MADS-M-type & 4 & 40 & up \\
\hline 11 & Ma09_g21260 & MADS-MIKC & 166 & 625 & up \\
\hline 12 & Ma11_g02670 & MADS-MIKC & 58 & 9 & down \\
\hline 13 & Ma11_g07440 & MADS-MIKC & 453 & 101 & down \\
\hline 14 & Ma02_g08090 & $\mathrm{SBP}$ & 106 & 49 & down \\
\hline 15 & Ma03_g10910 & SBP & 51 & 14 & down \\
\hline 16 & Ma04_g05770 & SBP & 143 & 51 & down \\
\hline 17 & Ma04_g12470 & SBP & 263 & 116 & down \\
\hline 18 & Ma05_g24390 & SBP & 201 & 86 & down \\
\hline 19 & Ma05_g25050 & SBP & 257 & 93 & down \\
\hline 20 & Ma06_g07650 & SBP & 10 & 1 & down \\
\hline 21 & Ma06_g24590 & SBP & 32 & 9 & down \\
\hline 22 & Ma08_g24570 & SBP & 833 & 189 & down \\
\hline 23 & Ma09_g16630 & SBP & 621 & 202 & down \\
\hline 24 & Ma09_g23570 & SBP & 52 & 20 & down \\
\hline 25 & Ma09_g28300 & SBP & 45 & 15 & down \\
\hline 26 & Ma11_g18010 & SBP & 144 & 52 & down \\
\hline 27 & Ma00_g01720 & NAC & 50 & 2 & down \\
\hline 28 & Ma02_g01890 & NAC & 595 & 187 & down \\
\hline 29 & Ma02_g10970 & NAC & 39 & 575 & up \\
\hline 30 & Ma03_g09370 & NAC & 276 & 1399 & up \\
\hline 31 & Ma04_g19710 & NAC & 1616 & 724 & down \\
\hline 32 & Ma05_g07350 & NAC & 258 & 49 & down \\
\hline 33 & Ma05_g07360 & NAC & 758 & 214 & down \\
\hline 34 & Ma05_g20080 & NAC & 18 & 2 & down \\
\hline 35 & Ma05_g20400 & NAC & 554 & 269 & down \\
\hline 36 & Ma05_g21000 & NAC & 9 & 0 & down \\
\hline 37 & Ma05_g29000 & NAC & 64 & 11 & down \\
\hline 38 & Ma06_g03480 & NAC & 9 & 56 & up \\
\hline 39 & Ma06_g19100 & NAC & 140 & 34 & down \\
\hline 40 & Ma06_g25140 & NAC & 135 & 620 & up \\
\hline 41 & Ma06_g27580 & NAC & 1189 & 376 & down \\
\hline 42 & Ma06_g28730 & NAC & 104 & 316 & up \\
\hline 43 & Ma06_g33980 & NAC & 114 & 6191 & up \\
\hline 44 & Ma07_g24800 & NAC & 5505 & 11,884 & up \\
\hline 45 & Ma07_g27560 & NAC & 3026 & 1430 & down \\
\hline 46 & Ma08_g09680 & NAC & 46 & 118 & up \\
\hline 47 & Ma09_g01160 & NAC & 797 & 52 & down \\
\hline 48 & Ma09_g01850 & NAC & 0 & 8 & up \\
\hline 49 & Ma09_g19410 & NAC & 200 & 14 & down \\
\hline
\end{tabular}


Table 1 (continued)

\begin{tabular}{|c|c|c|c|c|c|}
\hline Number & Gene ID & Family & $\begin{array}{l}\text { Young fruit } \\
\text { Expression level }\end{array}$ & $\begin{array}{l}\text { Mature green fruit } \\
\text { Expression level }\end{array}$ & regulated \\
\hline 50 & Ma09_g24910 & NAC & 144 & 45 & down \\
\hline 51 & Ma09_g28160 & NAC & 136 & 25 & down \\
\hline 52 & Ma09_g30350 & NAC & 26 & 73 & up \\
\hline 53 & Ma11_g01240 & NAC & 151 & 39 & down \\
\hline 54 & Ma11_g16350 & NAC & 111 & 15 & down \\
\hline 55 & Ma11_g20940 & NAC & 0 & 9 & up \\
\hline 56 & Ma11_g21100 & NAC & 71 & 24 & down \\
\hline 57 & Ma11_g24060 & NAC & 95 & 25 & down \\
\hline 58 & Ma00_g00100 & AP2/ERF-ERF & 29 & 88 & up \\
\hline 59 & Ma01_g17470 & AP2/ERF-AP2 & 52 & 10 & down \\
\hline 60 & Ma01_g20010 & AP2/ERF-AP2 & 15 & 1 & down \\
\hline 61 & Ma02_g00070 & AP2/ERF-ERF & 39 & 87 & up \\
\hline 62 & Ma02_g09470 & AP2/ERF-ERF & 205 & 52 & down \\
\hline 63 & Ma02_g17400 & AP2/ERF-ERF & 309 & 55 & down \\
\hline 64 & Ma02_g23280 & AP2/ERF-ERF & 300 & 77 & down \\
\hline 65 & Ma03_g04220 & AP2/ERF-ERF & 434 & 210 & down \\
\hline 66 & Ma03_g04940 & AP2/ERF-AP2 & 11 & 0 & down \\
\hline 67 & Ma03_g05830 & AP2/ERF-ERF & 6 & 0 & down \\
\hline 68 & Ma03_g08090 & AP2/ERF-ERF & 146 & 54 & down \\
\hline 69 & Ma03_g12670 & AP2/ERF-ERF & 593 & 2087 & up \\
\hline 70 & Ma03_g19980 & AP2/ERF-ERF & 17 & 2 & down \\
\hline 71 & Ma03_g23580 & AP2/ERF-ERF & 389 & 48 & down \\
\hline 72 & Ma04_g06130 & AP2/ERF-AP2 & 96 & 4 & down \\
\hline 73 & Ma04_g09020 & AP2/ERF-ERF & 2 & 60 & up \\
\hline 74 & Ma04_g09890 & AP2/ERF-ERF & 43 & 3 & down \\
\hline 75 & Ma04_g17170 & AP2/ERF-ERF & 735 & 319 & down \\
\hline 76 & Ma04_g20370 & AP2/ERF-ERF & 139 & 5 & down \\
\hline 77 & Ma04_g21170 & AP2/ERF-ERF & 48 & 629 & up \\
\hline 78 & Ma04_g26920 & AP2/ERF-ERF & 998 & 2708 & up \\
\hline 79 & Ma05_g04410 & AP2/ERF-AP2 & 239 & 1289 & up \\
\hline 80 & Ma05_g04880 & AP2/ERF-ERF & 21 & 110 & up \\
\hline 81 & Ma05_g26400 & AP2/ERF-ERF & 27 & 1 & down \\
\hline 82 & Ma05_g31650 & AP2/ERF-AP2 & 268 & 108 & down \\
\hline 83 & Ma06_g01950 & AP2/ERF-ERF & 411 & 934 & up \\
\hline 84 & Ma06_g09740 & AP2/ERF-ERF & 496 & 1590 & up \\
\hline 85 & Ma06_g15710 & AP2/ERF-ERF & 47 & 12 & down \\
\hline 86 & Ma06_g24790 & AP2/ERF-ERF & 206 & 636 & up \\
\hline 87 & Ma06_g36350 & AP2/ERF-AP2 & 86 & 242 & up \\
\hline 88 & Ma08_g01560 & AP2/ERF-ERF & 21 & 75 & up \\
\hline 89 & Ma08_g01810 & AP2/ERF-AP2 & 14 & 1 & down \\
\hline 90 & Ma08_g09060 & AP2/ERF-AP2 & 26 & 3 & down \\
\hline 91 & Ma08_g21180 & AP2/ERF-ERF & 52 & 1 & down \\
\hline 92 & Ma09_g03040 & AP2/ERF-AP2 & 427 & 1114 & up \\
\hline 93 & Ma09_g12570 & AP2/ERF-ERF & 698 & 207 & down \\
\hline 94 & Ma10_g01280 & AP2/ERF-AP2 & 51 & 17 & down \\
\hline 95 & Ma10_g01420 & AP2/ERF-ERF & 50 & 204 & up \\
\hline 96 & Ma10_g14680 & AP2/ERF-ERF & 13 & 0 & down \\
\hline 97 & Ma10_g19030 & AP2/ERF-ERF & 60 & 11 & down \\
\hline 98 & Ma10_g19470 & AP2/ERF-ERF & 604 & 2022 & up \\
\hline
\end{tabular}


Table 1 (continued)

\begin{tabular}{|c|c|c|c|c|c|}
\hline Number & Gene ID & Family & $\begin{array}{l}\text { Young fruit } \\
\text { Expression level }\end{array}$ & $\begin{array}{l}\text { Mature green fruit } \\
\text { Expression level }\end{array}$ & regulated \\
\hline 99 & Ma10_g21410 & AP2/ERF-ERF & 49 & 7 & down \\
\hline 100 & Ma10_g26420 & AP2/ERF-ERF & 19 & 3 & down \\
\hline 101 & Ma10_g31080 & AP2/ERF-ERF & 12 & 1 & down \\
\hline 102 & Ma11_g20400 & AP2/ERF-ERF & 815 & 63 & down \\
\hline 103 & Ma00_g01590 & MYB & 457 & 1798 & up \\
\hline 104 & Ma01_g02530 & MYB-related & 173 & 57 & down \\
\hline 105 & Ma01_g14370 & MYB & 1367 & 404 & down \\
\hline 106 & Ma01_g17260 & MYB & 49 & 21 & down \\
\hline 107 & Ma01_g17870 & MYB-related & 117 & 284 & up \\
\hline 108 & Ma01_g19610 & MYB & 280 & 14 & down \\
\hline 109 & Ma02_g01300 & MYB-related & 154 & 20 & down \\
\hline 110 & Ma02_g05880 & MYB & 145 & 50 & down \\
\hline 111 & Ma02_g09720 & MYB & 19 & 0 & down \\
\hline 112 & Ma02_g09870 & MYB & 29 & 1 & down \\
\hline 113 & Ma02_g10870 & MYB-related & 8 & 0 & down \\
\hline 114 & Ma02_g17950 & MYB & 47 & 10 & down \\
\hline 115 & Ma02_g19770 & MYB & 5 & 27 & up \\
\hline 116 & Ma03_g07840 & MYB & 18 & 0 & down \\
\hline 117 & Ma03_g12720 & MYB & 344 & 1201 & up \\
\hline 118 & Ma03_g25780 & MYB & 47 & 11 & down \\
\hline 119 & Ma04_g12940 & MYB & 138 & 47 & down \\
\hline 120 & Ma04_g24670 & MYB & 0 & 6 & up \\
\hline 121 & Ma04_g26220 & MYB & 37 & 4 & down \\
\hline 122 & Ma05_g07450 & MYB & 46 & 1 & down \\
\hline 123 & Ma05_g08940 & MYB-related & 8 & 0 & down \\
\hline 124 & Ma05_g12030 & MYB & 86 & 7 & down \\
\hline 125 & Ma05_g23640 & MYB & 33 & 0 & down \\
\hline 126 & Ma05_g30120 & MYB & 107 & 19 & down \\
\hline 127 & Ma06_g04270 & MYB & 106 & 42 & down \\
\hline 128 & Ma06_g08910 & MYB & 198 & 88 & down \\
\hline 129 & Ma06_g11140 & MYB & 3 & 27 & up \\
\hline 130 & Ma06_g11270 & MYB & 39 & 9 & down \\
\hline 131 & Ma06_g12110 & MYB & 142 & 62 & down \\
\hline 132 & Ma06_g12160 & MYB & 45 & 102 & up \\
\hline 133 & Ma06_g16920 & MYB & 170 & 51 & down \\
\hline 134 & Ma06_g20700 & MYB-related & 5 & 20 & up \\
\hline 135 & Ma06_g33920 & MYB & 1 & 15 & up \\
\hline 136 & Ma07_g05780 & MYB & 87 & 272 & up \\
\hline 137 & Ma07_g19720 & MYB & 134 & 49 & down \\
\hline 138 & Ma07_g19880 & MYB & 58 & 24 & down \\
\hline 139 & Ma07_g23180 & MYB & 33 & 5 & down \\
\hline 140 & Ma07_g23230 & MYB & 840 & 3573 & up \\
\hline 141 & Ma07_g27070 & MYB-related & 2 & 13 & up \\
\hline 142 & Ma08_g01760 & MYB & 53 & 438 & up \\
\hline 143 & Ma08_g02180 & MYB & 526 & 1196 & up \\
\hline 144 & Ma08_g14720 & MYB & 37 & 11 & down \\
\hline 145 & Ma08_g15820 & MYB & 47 & 10 & down \\
\hline 146 & Ma08_g23390 & MYB & 248 & 1 & down \\
\hline 147 & Ma08_g25960 & MYB & 173 & 63 & down \\
\hline
\end{tabular}


Table 1 (continued)

\begin{tabular}{|c|c|c|c|c|c|}
\hline Number & Gene ID & Family & $\begin{array}{l}\text { Young fruit } \\
\text { Expression level }\end{array}$ & $\begin{array}{l}\text { Mature green fruit } \\
\text { Expression level }\end{array}$ & regulated \\
\hline 148 & Ma09_g04930 & MYB & 62 & 2 & down \\
\hline 149 & Ma09_g05760 & MYB-related & 199 & 41 & down \\
\hline 150 & Ma09_g20610 & MYB & 537 & 239 & down \\
\hline 151 & Ma09_g25590 & MYB & 8 & 0 & down \\
\hline 152 & Ma09_g28270 & MYB-related & 239 & 608 & up \\
\hline 153 & Ma09_g30920 & MYB-related & 363 & 164 & down \\
\hline 154 & Ma10_g06750 & MYB-related & 49 & 165 & up \\
\hline 155 & Ma10_g14950 & MYB & 12 & 1 & down \\
\hline 156 & Ma10_g16050 & MYB & 125 & 22 & down \\
\hline 157 & Ma10_g26660 & MYB & 35 & 1 & down \\
\hline 158 & Ma11_g01360 & MYB-related & 77 & 17 & down \\
\hline 159 & Ma11_g03860 & MYB & 18 & 1 & down \\
\hline 160 & Ma11_g10680 & MYB & 725 & 12 & down \\
\hline 161 & Ma11_g14670 & MYB & 43 & 194 & up \\
\hline 162 & Ma11_g16430 & MYB & 91 & 23 & down \\
\hline 163 & Ma03_g11720 & NF-YC & 35 & 75 & up \\
\hline 164 & Ma04_g34950 & NF-YA & 127 & 45 & down \\
\hline 165 & Ma04_g38010 & NF-YA & 49 & 12 & down \\
\hline 166 & Ma07_g01080 & NF-YA & 474 & 149 & down \\
\hline 167 & Ma07_g13230 & NF-YC & 39 & 89 & up \\
\hline 168 & Ma08_g18750 & NF-YA & 29 & 8 & down \\
\hline 169 & Ma08_g22650 & NF-YB & 0 & 9 & up \\
\hline 170 & Ma11_g23990 & NF-YC & 43 & 8 & down \\
\hline
\end{tabular}

development $[28,29]$. This finding was consistent with the obtained results that most of the carotenoid compounds were undetectable or at considerably low levels at young fruits but markedly increased at the mature green and/or mature fruits. These results all suggest that the synthesis of carotenoids mainly occurs in the middle and late stages of fruit development $[28,29]$.

RNA sequencing of the samples at three critical developmental stages was performed to understand the genome-wide expression patterns during fruit development. A large number of DEGs across the samples revealed a stage-specific transcriptome profile during fruit development [30]. The GO analysis classified 18,839, 17,800 , and 17,469 genes into the biological process, cell component, and molecular function, respectively. These function annotations demonstrated that the gene expressed in banana encodes diverse metabolism-related proteins [23]. KEGG analysis revealed that DEGs were mainly involved in the biosynthesis of secondary metabolites, arachidonic acid metabolism, plant hormone signal transduction, and endocrine and other factor-regulated calcium reabsorption. This study focused on differential carotenoid accumulation during fruit development. The carotenoid accumulation in plants is a complex process associated with the expression of genes involved in carotenoid biosynthesis, degradation, and storage [31]. Carotenoid biosynthesis was enriched in the comparison of young and mature green fruits. Seven DEGs involved in carotenoid biosynthesis were identified, suggesting that these genes may be responsible for the differential carotenoid accumulation during fruit development. A putative road map of carotenoid biosynthesis was also drawn. Notably, most of the DEGs gradually increased with fruit development, which is consistent with the carotenoid metabolic characteristics discussed above and the previous reports $[28,32]$. In the current study, the gene encoding Z-ISO gradually increased with fruit development, which is directly correlated with the accumulation of lycopene [28].

The expression of gene encoding lycopene $\beta$-cyclase (LCYB), lycopene $\varepsilon$-cyclase (LCYE), and $\beta$-carotene hydroxylase gradually increased with fruit development to verify the high contents of carotenoid at the middle and late stages of fruit development. Moreover, the expression level of the gene encoding violaxanthin de-epoxidase (VDE) gradually decreased with fruit development, which resulted in the low content of violaxanthin in mature green and mature fruits. These results 


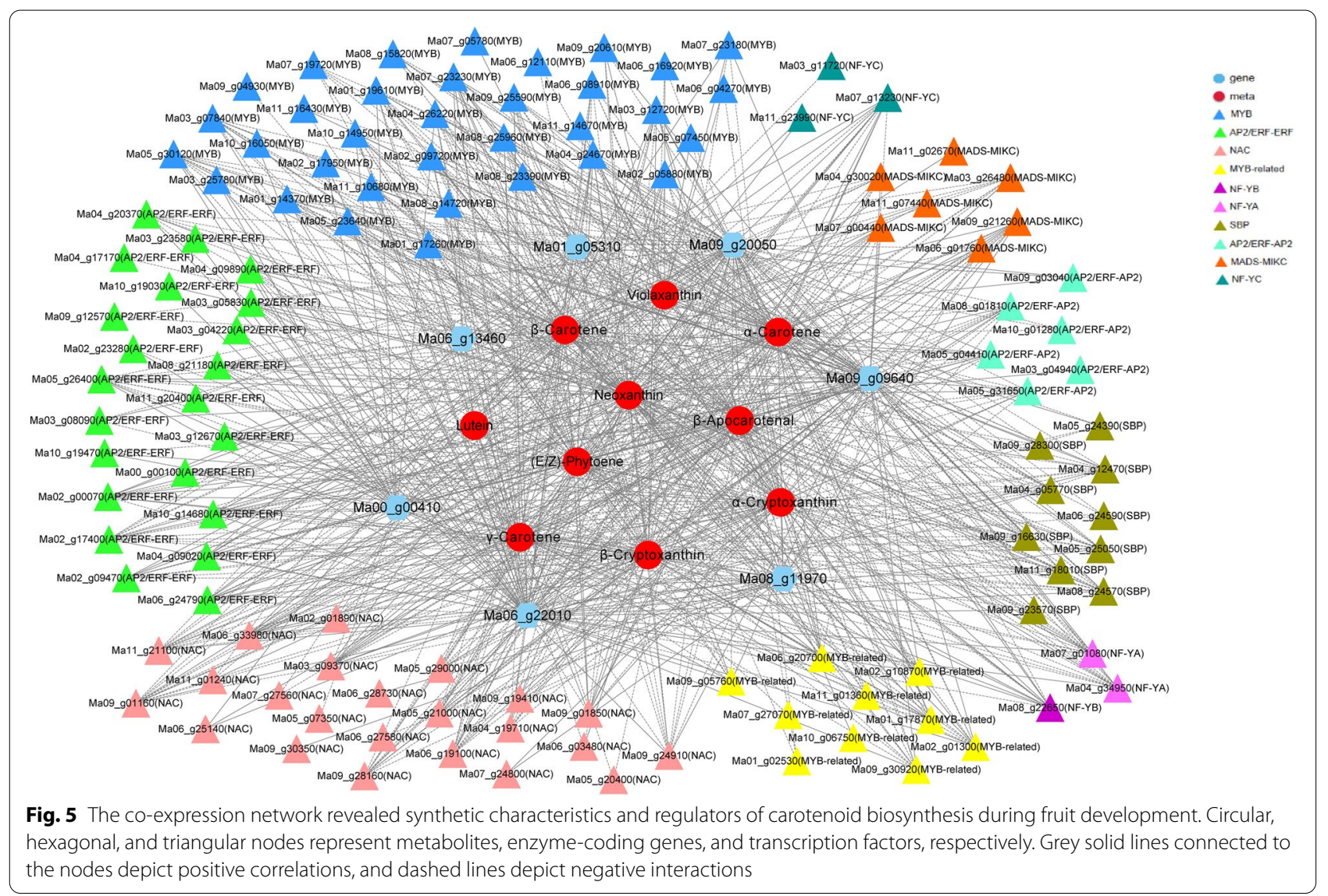

suggested that the content of carotenoids is closely related to the expression of structural genes [33].

The transcriptional regulation of carotenoid biosynthetic genes is the first level and an important control mechanism for carotenoid production in fruits [34]. Transcription factors are critical for the regulation of these biosynthetic gene expressions. LCYB is crucial in branching the metabolic flux into either $\alpha$-carotene in $\beta, \varepsilon$-branch or $\beta$-carotene in $\beta, \beta$-branch of the pathway [34-36]. In the present study, co-expression network analysis revealed that 79 differentially expressed transcription factor genes may be responsible for the regulation of LCYB. The functional analysis of these DEGs will contribute to the understanding regarding the molecular mechanism of carotenoid accumulation in bananas.

\section{Conclusion}

The mechanisms of carotenoid accumulation during banana fruit development were analyzed in this study by using the dynamic metabolites, transcriptome, and qRTPCR. A total of 11 carotenoid compounds were identified, and most of these compounds had high contents of carotenoid at the middle and late stages of fruit development. Furthermore, a series of carotenoid biosynthetic and regulatory genes were analyzed by RNA-seq and qRT-PCR . Collectively, these findings provide new information on the mechanisms of carotenoid accumulation during banana fruit development and a series of candidate genes with applications in the breeding of special banana subgroups with high carotenoid contents. It is difficult to improve fruit quality by conventional breeding, however molecular breeding which uses gene editing technology might breed directionally high carotenoid content of banana.

\section{Methods}

\section{Plant materials and treatment}

The Xiangfen1 banana plants used in this study were planted in an orchard at South Subtropical Crop Research Institute, Chinese Academy of Tropical Agricultural Science, Zhanjiang, Guangdong, China $\left(21^{\circ} 27 \mathrm{~N}, 110^{\circ} 35^{\prime} \mathrm{E}\right)$. Xiangfen1 banana fruit samples at three different developmental stages (cut off flower days 45, 85, and $85+3$ ) were collected from the banana plantation. The fruits collected on the 3 days (days 45,85 , and $85+3$ ) represented three typical samples of banana (young, mature green, and mature fruits, respectively). All flesh samples were immediately frozen in liquid nitrogen and stored at $-80^{\circ} \mathrm{C}$ until further use. 


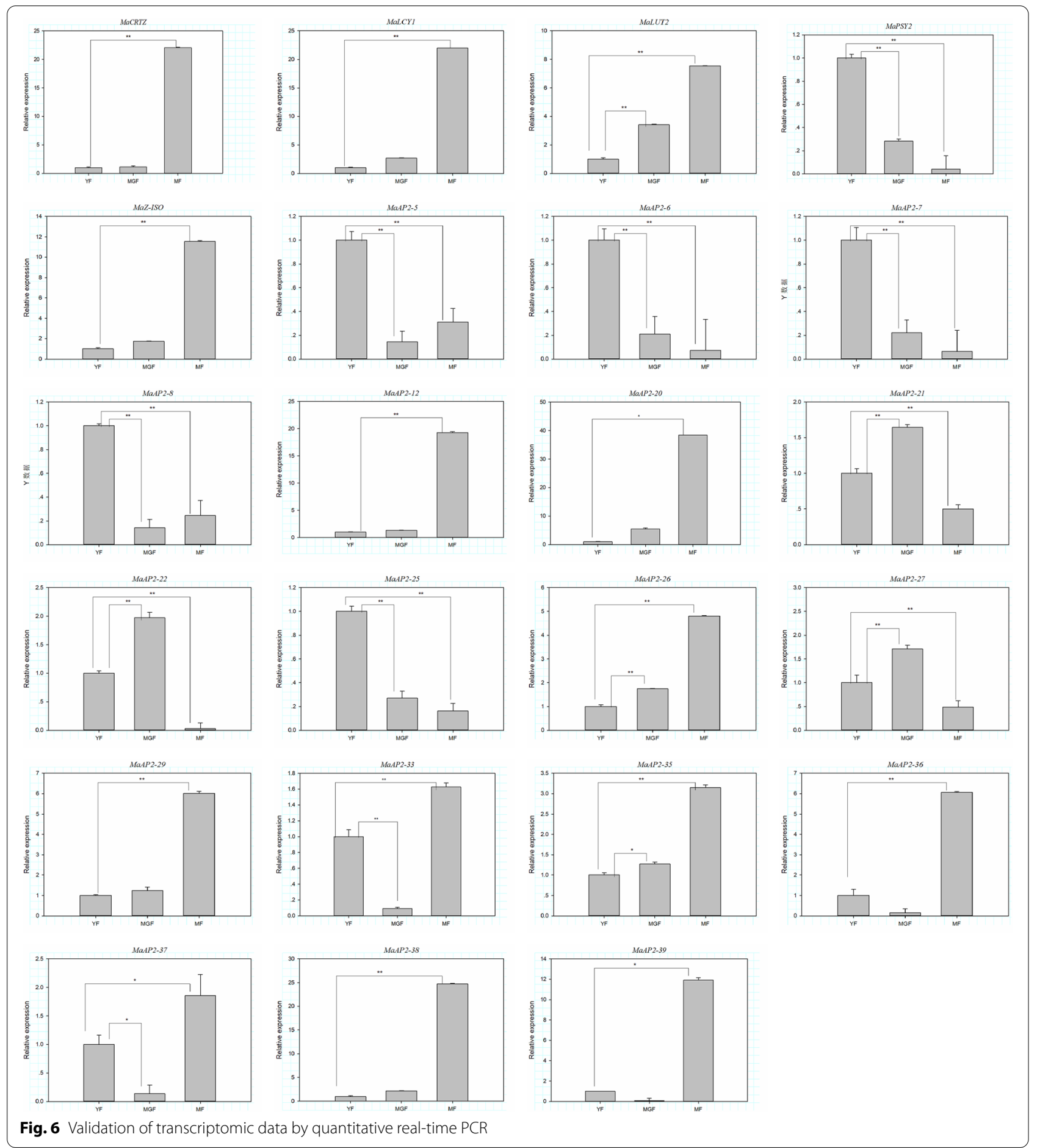

\section{Chemicals and reagents}

Methanol $(\mathrm{MeOH})$, Ethanol (EtOH), Acetone, Methyl tert-butyl ether and BHT were purchased from Merck (Darmstadt, Germany). MilliQ water (Millipore, Bradford, USA) was used in all experiments. All of the standards were purchased from Olchemim Ltd. (Olomouc, Czech Republic) and Sigma (St. Louis, MO, USA). Formic acid was obtained from Sigma. The stock solutions of standards were prepared at the concentration of $1 \mathrm{mg} / \mathrm{mL}$. All stock solutions were stored at $-20^{\circ} \mathrm{C}$. 


\section{Sample preparation and extraction}

Fresh plant materials were freeze dried, and stored at $-80^{\circ} \mathrm{C}$ until needed. All analyses were performed in triplicate. Then dried plant materials were homogenized and powdered in a mill. $50 \mathrm{mg}$ of dried powder was extracted with mixed solution of $n$-hexane: acetone: ethanol, and add internal standard. The extract was vortexed for $20 \mathrm{~min}$ at room temperature. The supernatants were collected after centrifugation. The residue was re-extracted and repeat the steps above. Both supernatants were collected and then evaporated to dryness under nitrogen gas stream, reconstituted in mixed solution of methanol: MTBE. The solution was filtered through $0.22 \mu \mathrm{m}$ filter for further LC-MS analysis [37].

\section{HPLC conditions}

The sample extracts were analyzed using an LC- APCIMS/MS system (UHPLC, ExionLC AD, https://sciex.com. cn/; MS, Applied Biosystems 6500 Triple Quadrupole, https://sciex.com.cn/). The analytical conditions were as follow, HPLC: column, YMC C30 $(3 \mu \mathrm{m}, 2 \mathrm{~mm} * 100 \mathrm{~mm})$; solvent system, methanol: acetonitrile $(3: 1, \mathrm{~V} / \mathrm{V})(0.01 \%$ BHT, 0.1\% formic acid): methyl tert-butyl ether $(0.01 \%$ BHT); gradient program, $100: 0 \mathrm{~V} / \mathrm{V}$ at $0 \mathrm{~min}, 100: 0 \mathrm{~V} / \mathrm{V}$ at $3 \mathrm{~min}, 58: 42 \mathrm{~V} / \mathrm{V}$ at $6 \mathrm{~min}, 20: 80 \mathrm{~V} / \mathrm{V}$ at $8 \mathrm{~min}, 5: 95 \mathrm{~V} / \mathrm{V}$ at $9 \mathrm{~min}, 100: 0 \mathrm{~V} / \mathrm{V}$ at $9.1 \mathrm{~min}, 100: 0 \mathrm{~V} / \mathrm{V}$ at $11 \mathrm{~min}$; flow rate, $0.8 \mathrm{~mL} / \mathrm{min}$; temperature, $28^{\circ} \mathrm{C}$; injection volume: $2 \mu \mathrm{L}$ [38].

\section{APCl-q trap-MS/MS}

API 6500 Q TRAP LC/MS/MS System, equipped with an APCI Turbo Ion-Spray interface, operating in a positive ion mode and controlled by Analyst 1.6.3 software (AB Sciex). The APCI source operation parameters were as follow: ion source, APCI+; source temperature $350^{\circ} \mathrm{C}$; curtain gas (CUR) were set at $25.0 \mathrm{psi}$; the collision gas (CAD) was medium. DP and CE for individual MRM transition was done with further DP and CE optimization. A specific set of MRM transitions were monitored for each period according to the carotenoids eluted within this period [39].

\section{Detection of carotenoids}

$\alpha$-Carotene, $\beta$-Carotene, $\gamma$-Carotene, $\varepsilon$-Carotene, Lutein, Violaxanthin, Antheraxanthin, Neoxanthin, Zeaxanthin, $\beta$-Cryptoxanthin, $\alpha$-Cryptoxanthin, all-trans-Lycopene, Phytofluene, (E/Z)-Phytoene, Astaxanthin, Capsanthin, Apocarotenal and Capsorubin contents were detected by MetWare (http://www.metware.cn/) based on the AB Sciex QTRAP6500 LC-MS/MS platform.

\section{RT-qPCR validation}

RT-qPCR was applied to investigate gene expression patterns. First-strand cDNA was generated from $1 \mu \mathrm{g}$ total RNA isolated from the seven pericarp samples using the PrimeScript ${ }^{\mathrm{TM}} \mathrm{RT}$ reagent kit (TaKaRa, Japan). RT-qPCR primers were designed using Primer Premier 5.0 software (Premier, Canada) and synthesized by Sangon Biotech (Shanghai, China) Co., Ltd. The relative expression level of the genes were calculated using Eq. $2^{-\Delta \Delta C \mathrm{t}}$.

\section{Statistical analysis}

To reduce the dimension of data and simplify transcriptome data, principal component analysis (PCA), a multivariate statistical analysis method, was used in this study. The differential metabolites and genes were annotated using the Kyoto Encyclopedia of Genes and Genomes (KEGG) Pathway database (http://www.kegg.jp/kegg/ pathway.html).

\section{Abbreviations}

HPLC: High-performance liquid chromatography; UHPLC: Ultra high-performance liquid chromatography; LC-MS: Liquid chromatography-tandem mass spectrometry; IPP: Isopentenyl diphosphate; DMAPP: Dimethylallyl diphosphate; GGPP: Geranylgeranyl pyrophosphates; PCA: Principal component analysis.

\section{Acknowledgments}

Thanks for the South Subtropical Crop Research Institute, Chinese Academy of Tropical Agricultural Science.

\section{Authors' contributions}

JHX and HGH conceived and designed the experiments; CD, JXW, YLH and WJX performed the experiments and helped with the data analysis; and $\mathrm{HGH}$ and CD wrote the paper. All authors read and approved the final manuscript.

\section{Funding}

This work was supported by Natural Science Foundation of Guangdong Province (2020A1515010166) and National Key R\&D Program of China (Grant No. 2019YFD1000903).

\section{Availability of data and materials}

The datasets generated during the current study are available from the corresponding author on reasonable request. All raw read data were deposited in the Sequence Read Archive (SRA) in NCBI with the Bioproject ID: PRJNA776816 (https://dataview.ncbi.nlm.nih.gov/object/PRJNA776816?reviewer=ba2olltnfd 86gmonebc83286av).

\section{Declarations}

Ethics approval and consent to participate

All the materials of this project are given by South Subtropical Crop Research Institute, Chinese Academy of Tropical Agricultural Science (Zhanjiang,China). The experimental research on plants performed in this study complies with institutional, national and international guidelines.

\section{Consent for publication}

Not applicable.

\section{Competing interests}

The authors declare that they have no competing interests. 
Received: 4 November 2021 Accepted: 27 December 2021

Published online: 17 January 2022

\section{References}

1. Zhuang J, Hou C, Tang Y, He Y, Luo S. Assessment of external properties for identifying Banana fruit maturity stages using optical imaging techniques. Sensors. 2019;19(13):2910. https://doi.org/10.3390/s1913 2910.

2. Hong Z, Jiali Y, Yueming J, Jun Z, Xuesong Z, Yanglin H, et al. Morin as a preservative for delaying senescence of Banana. Biomolecules. 2018;8(3):52. https://doi.org/10.3390/biom8030052.

3. Lim YY, Lim TT, Tee JJ. Antioxidant properties of several tropical fruits: a comparative study. Food Chem. 2007;103(3):1003-8. https://doi.org/10. 1016/j.foodchem.2006.08.038.

4. Nisar N, Li L, Lu S, Khin NC, Pogson BJ. Carotenoid metabolism in plants. Mol Plant. 2015;8(1):68-82. https://doi.org/10.1016/j.molp.2014. 12.007

5. Rolland NCGF. The biosynthetic capacities of the plastids and integration between cytoplasmic and chloroplast processes. Annu Rev Genet. 2012;46(1):233-64. https://doi.org/10.1146/annur ev-genet-1 10410-132544.

6. Liu L, Shao Z, Zhang M, Wang Q. Regulation of carotenoid metabolism in tomato. Mol Plant. 2015;8(1):28-39. https://doi.org/10.1016/j.molp. 2014.11.006.

7. Yuan H, Zhang J, Nageswaran D, Li L. Carotenoid metabolism and regulation in horticultural crops. Hortic Res. 2015;2(15036):15036. https:// doi.org/10.1038/hortres.2015.36.

8. Llorente B. Regulation of carotenoid biosynthesis in photosynthetic organs: Springer International Publishing; 2016. https://doi.org/10. 1007/978-3-319-39126-7_5

9. Vogel JT, Tieman DM, Sims CA, Odabasi AZ, Clark DG, Klee HJ. Carotenoid content impacts flavor acceptability in tomato (Solanum lycopersicum). J Sci Food Agric. 2010;90(13):2233-40. https://doi.org/ 10.1002/jsfa.4076.

10. Fraser PD, Bramley PM. The biosynthesis and nutritional uses of carotenoids. Prog Lipid Res. 2004;43(3):228-65. https://doi.org/10.1016/j. plipres.2003.10.002.

11. Chappell J, Wolf F, Proulx J, Saunders CC. Is the reaction catalyzed by 3-Hydroxy-3-Methylglutaryl coenzyme a reductase a rate-limiting step for isoprenoid biosynthesis in plants? Plant Physiol. 1995;109(4):133743. https://doi.org/10.1104/pp.109.4.1337.

12. Rodriguez-Concepcion M. Elucidation of the methylerythritol phosphate pathway for isoprenoid biosynthesis in bacteria and plastids. A metabolic milestone achieved through genomics. Plant Physiol. 2002;130(3):1079-89. https://doi.org/10.1104/pp.007138.

13. Eisenreich W, Bacher A, Arigoni D, Rohdich F. Biosynthesis of isoprenoids via the non-mevalonate pathway. Cell Mol Life Sci. 2004;61 (12):1401. https://doi.org/10.1007/s00018-004-3381-z.

14. Hirschberg J. Carotenoid biosynthesis in flowering plants. Curr Opin Plant Biol. 2001;4. https://doi.org/10.1016/S1369-5266(00)00163-1.

15. Li L, Wang X, Zhang X, Guo M, Liu T. Unraveling the target genes of RIN transcription factor during tomato fruit ripening and softening. J Sci Food Agric. 2017:97(3):991-1000. https://doi.org/10.1002/jsfa.7825.

16. Salinas M, Xing S, Höhmann S, Berndtgen R, Huijser P. Genomic organization, phylogenetic comparison and differential expression of the SBP-box family of transcription factors in tomato. Planta. 2012;235(6):1171-84. https://doi.org/10.1007/s00425-011-1565-y.

17. Zhu M, Chen G, Shuang Z, Yun T, Yi W, Dong T, et al. A new tomato NAC (NAM/ATAF1/2/CUC2) transcription factor, SINAC4, functions as a positive regulator of fruit ripening and carotenoid accumulation. Plant Cell Physiol. 2014;1:119-35. https://doi.org/10.1093/pcp/pct162.

18. Je MLJJ. Combined transcriptome, genetic diversity and metabolite profiling in tomato fruit reveals that the ethylene response factor SIERF6 plays an important role in ripening and carotenoid accumulation. Plant J. 2012;70(2):191-204. https://doi.org/10.1111/j.1365-313X. 2011.04863.x

19. Nguyen CV, Vrebalov JT, Gapper NE, Zheng Y, Zhong S, Fei Z, et al. Tomato GOLDEN2-LIKE transcription factors reveal molecular gradients that function during fruit development and ripening. Plant Cell. 2014;26(2):585. https://doi.org/10.1105/tpc.113.118794.

20. Lin Z, Hong Y, Yin M, Li C, Zhang K, Grierson D. A tomato HD-zip homeobox protein, LeHB-1, plays an important role in floral organogenesis and ripening. Plant J. 2010;55. https://doi.org/10.1111/j.1365-313X 2008.03505.x.

21. Li S, Li K, Ju Z, Cao D, Fu D, Zhu H, et al. Genome-wide analysis of tomato NF-Y factors and their role in fruit ripening. BMC Genomics. 2016;17. https://doi.org/10.1186/s12864-015-2334-2 J, AU.

22. Ziran W, Yuanyuan C, Alexander V, Shangwu C, Huiqin M. Regulation of fig (Ficus carica L.) fruit color: Metabolomic and transcriptomic analyses of the flavonoid biosynthetic pathway. Frontiers. Plant Sci. 2017;8:1990. https://doi.org/10.1038/s41396-017-0004-X.

23. Dong T, Han R, Yu J, Zhu M, Zhang Y, Gong Y, et al. Anthocyanins accumulation and molecular analysis of correlated genes by metabolome and transcriptome in green and purple asparaguses ( Asparagus officinalis , L.). Food Chem. 2018;271(JAN.15):18-28. https://doi.org/10. 1016/j.foodchem.2018.07.120.

24. Wang $Y$, Zhang $X$, Yang S, Yuan $Y$. Lignin involvement in programmed changes in peach-fruit texture indicated by metabolite and transcriptome analyses. J Agr Food Chem. 2018;66(48):12627-40. https://doi. org/10.1021/acs.jafc.8b04284.

25. Jie M, Wang B, He G, Xianfeng T, Wang S, Ma Y, et al. Metabolomics Integrated with Transcriptomics Reveals Redirection of the Phenylpropanoids Metabolic Flux in Ginkgo biloba. J Agr Food Chem. 2019. https://doi.org/10.1021/acs.jafc.8b06355.

26. Du M, Li Y, Xie P, Gao YXPY. Nutritional component analyses of kiwifruit in different development stages by metabolomic and transcriptomic approaches. J Sci Food Agr. 2020;100(6):2399-409. https://doi.org/10. 1002/jsfa.10251.

27. Rodríguez-Rodríguez E, Sánchez-Prieto M, Olmedilla BOO. Assessment of carotenoid concentrations in red peppers (Capsicum annuum) under domestic refrigeration for three weeks as determined by HPLCDAD. Food Chem. 2020;6:100092. https://doi.org/10.1016/j.fochx.2020. 100092.

28. Heng Z, Sheng O, Huang W, Zhang S, Fernie AR, Motorykin I, et al. Integrated proteomic and metabolomic analysis suggests high rates of glycolysis are likely required to support high carotenoid accumulation in banana pulp. Food Chem. 2019;297(NOV.1):125011-6. https://doi. org/10.1016/j.foodchem.2019.125016.

29. Yla B, Jla B, Zla B, Jing WA, By B, Wc B, et al. Integrative analysis of metabolome and transcriptome reveals the mechanism of color formation in pepper fruit ( Capsicum annuum L.). Food Chem. 2019;306:125629. https://doi.org/10.1016/j.foodchem.2019.125629.

30. Hao Z, Liu S, Hu L, Shi J, Chen J. Transcriptome analysis and metabolic profiling reveal the key role of carotenoids in the petal coloration of Liriodendron tulipifera. Hortic Res. 2020;7(1).10.1038/ s41438-020-0287-3.

31. Cazzonelli Cl, Pogson BJ. Source to sink: regulation of carotenoid biosynthesis in plants. Trends Plant Sci. 2010;15(5):266-74. https://doi.org/ 10.1016/j.tplants.2010.02.003

32. Hexin $\tan X C N L$ : transcriptome analysis reveals novel enzymes for apocarotenoid biosynthesis in saffron and allows construction for crocetin synthesis in yeast. J Exp Bot. 2019;70(18):4819-34. https://doi.org/10. 1093/jxb/erz211.

33. Alos E, Martinez-Fuentes A, Reig C, Mesejo C, Zacarías L, Agustí M, et al. Involvement of ethylene in color changes and carotenoid biosynthesis in loquat fruit (Eriobotrya japonica Lindl. cv. Algerie). Postharvest Biol Tec. 2019;149:129-38. https://doi.org/10.1016/j.postharvbio.2018.11. 022.

34. Tsa B, Li L. Toward the 'golden' era: the status in uncovering the regulatory control of carotenoid accumulation in plants - ScienceDirect. Plant Sci. 2019;290:110331. https://doi.org/10.1016/j.plantsci.2019.110331.

35. Bai L, Kim EH, Dellapenna D, Brutnell TP. Novel lycopene epsilon cyclase activities in maize revealed through perturbation of carotenoid biosynthesis. Plant J. 2009;59(4):588-99. https://doi.org/10.1111/j.1365-313X. 2009.03899.x.

36. Giorio G, Yildirim A, Stigliani AL. D Ambrosio C: elevation of lutein content in tomato: a biochemical tug-of-war between lycopene cyclases. Metab Eng. 2013;20:167-76. https://doi.org/10.1016/j.ymben.2013.10. 007. 
37. Zhou W, Niu Y, Ding X, Zhao S, Liao K. Analysis of carotenoid content and diversity in apricots (Prunus armeniaca L.) grown in China. Food Chem. 2020;330:127223. https://doi.org/10.1016/j.foodchem.2020. 127223.

38. Petry FC, Mercadante AZ. New method for carotenoid extraction and analysis by HPLC-DAD-MS/MS in freeze-dried Citrus and mango pulps. J Brazil Chem Soc. 2018. https://doi.org/10.21577/0103-5053.20170127.

39. Yan N, Du Y, Liu X, Chu M, Shi J, Zhang H, et al. A comparative UHPLCQqQ-MS-based metabolomics approach for evaluating Chinese and north American wild rice. Food Chem. 2019;275(MAR.1):618-27. https://doi.org/10.1016/j.foodchem.2018.09.153.

\section{Publisher's Note}

Springer Nature remains neutral with regard to jurisdictional claims in published maps and institutional affiliations.

- fast, convenient online submission

- thorough peer review by experienced researchers in your field

- rapid publication on acceptance

- support for research data, including large and complex data types

- gold Open Access which fosters wider collaboration and increased citations

- maximum visibility for your research: over 100M website views per year

At BMC, research is always in progress.

Learn more biomedcentral.com/submissions 